\title{
Quasilinearization and the Estimation of Chemical Rate Constants from Raw Kinetic Data*
}

RICHARD BELLMAN, University of Southern California, Los Angeles, J. JACQUEZ. University of Michigan, Ann Arbor,

R. KALABA AND S. SCHWIMMER, The RAND Corporation, Santa Monica, California

Communicated sy Richard Bellman

\begin{abstract}
A technique for estimating chemical rate constants from raw kinetic data is suggested. Such problems are viewed as nonlinear multipoint boundary-value problems for systems of noniinear ordinary differential equations, for which the quasilinearization procedure offers an effective means of numerical solution. The method is illustrated using kinetic data obtained by Bodenstein and Lindner on some gas phase reactions of nitrogen and oxygen.
\end{abstract}

\section{INTRODUCTION}

Recent advances in the technology of digital computers have made the numerical solution of large systems of nonlinear differential equations, subject to initial conditions, a routine affair. With this basis, many effective techniques can be devised for the numerical solution of two-point and multipoint boundary-value problems [1]. This has far-reaching implications for science and technology, from both theoretical and experimental aspects.

The reason for this may be briefly described as follows. Theory frequently provides equations describing a process without giving the numerical values of constants appearing in the equations. Observationss

\footnotetext{
* This investigation was supported in part by Public Health Service research grant GM-09608-04 from the Division of General Medical Sciences, National Institutes of Health.
} 
and experiments are then used to provide data for the determination of the unknown parameters. This reconciliation of therry and experiment is of basic importance, since it is a fundarnental method for testing the validity of cause-and-effect assumptions and of theoratical structures in general. Many techniques are available for reduling the numerical solutions of more complex functional equations involving partial derivatives and integrals to the solution of systems of ordinary differential equations (see [2-6]).

In this paper we indicate the application of the general method of quasilinearization to the estimation of chemical rate constants using raw kinetic data. Bodenstein and Lindner [7] have presented data on pressures observed during the reversible homogeneous gas phase reaction

$$
2 \mathrm{NO}+\mathrm{O}_{2} \rightleftharpoons 2 \mathrm{NO}_{2} \text {. }
$$

The experiments were carried out at constant volume and temperature. The forward reaction is third order and the backward reaction is second order. The descriptive differential equation, after various normalizations, has the form

$$
\frac{d x}{d t}=k(a-x)(b-x)^{2}-K x^{2},
$$

where $a=126.2$ and $b=91.9$, and $k$ and $K$ are the rate constants to be estimated on the basis of the observations given in Table I.

\section{TABLE I}

EXPERIMENTAL DATA ${ }^{a}$

\begin{tabular}{cccccccccccccccc}
\hline$t$ & 0 & 1 & 2 & 3 & 4 & 5 & 6 & 7 & 9 & 11 & 14 & 19 & 24 & 29 & 39 \\
$x$ & 0 & 1.4 & 6.3 & 10.5 & 14.2 & 17.6 & 21.4 & 23.0 & 27.0 & 30.5 & 34.4 & 48.8 & 41.6 & 43.5 & 45.3 \\
\hline
\end{tabular}

a Table 39 in Bodenstein and Lindner [7].

Let us denote the $n$th observation at tirne $t_{n}$ by $w_{n}, t_{1}=1, w_{1}=1.4$, and so on. We then want to determine the rate constants $k$ and $K$ so that the solution of the differential Eq. 2, subject to the initial condition $x(0)=0$, will agree as closely as porsible with the measured values. We must, of corrse, intrởuce an appropriate measure of closeness. 
Quasilinearization provides a successive approximation method for solving the estimation pro?lem just posed. It is a quadratically convergent technique, so that if we begin with a reasonably good initial estimate, we can expect to double the number of correct digits asymptotically with each additional step in the process. Here, asymptoticaily usually means from the third iteration on.

We can first transform the problem of estimating constants within the equation into one in which we estimate the initial conditions for a system of differential equations. We do this by considering $k$ and $K$ to be functions of time that satisfy the differential equations

$$
\begin{aligned}
& \dot{k}=0, \\
& \dot{K}=\mathbf{0} .
\end{aligned}
$$

The aim is to consider all quantities as functions of time, and all unknowns appear as initial conditions.

As a measure of closeness, we now minimize the quantity

$$
Q=\sum_{i=1}^{14}\left(x\left(t_{i}\right)-w_{i}\right)^{2}, \quad i
$$

where

$$
\begin{aligned}
\dot{x} & =k(126.2-x)(91.9-x)^{2}-K x^{2}, \quad x(0)=0, \\
k & =0, \\
\dot{K} & =0 .
\end{aligned}
$$

The minimization is over the initial values of $k$ and $K, k(0)$ and $K(0)$.

We will sketch the quasilinearization technique in quite general terms. Let the $N$-dimensional vector $x(t)$ be a solution of the differential equation

$$
\dot{x}=f(x), \quad x_{i}(0)=\alpha_{i}, \quad i=1,2, \ldots, S .
$$

The first $S$ components of $x(0)$ are specified, and the last $N-S$ are free to be chosen so as to minimize the sum

$$
Q=\sum_{i=1}^{M}\left[\left(\beta, x\left(t_{i}\right)\right)-w_{i}\right]^{2}, \quad M \geqslant N-S .
$$


As usual, $(\beta, x)$ denotes an inner product, and $\beta$ is a constant weighting vector. As was remarked earlier, our procedure is an iterative one. First we select an initial approximation to the missing initial conditions and integrate Eq. 9 numerically on the interval $0 \leqslant t \leqslant t_{m}$. Call this vector function $x^{0}(t)$. Next we corisider a linearized version of Eq. 9

$$
\dot{x}^{1}=f\left(x^{0}\right)+J\left(x^{0}\right)\left(x^{1}-x^{0}\right)
$$

where the Jacobian matrix $J$ is given by

$$
J\left(x^{0}\right)=\frac{\partial f_{i}\left(x^{0}\right)}{\partial x_{j}} .
$$

This matrix is known computationally on the interval $0 \leqslant t \leqslant t_{m}$. We produce numericaliy a particular solution $p(t)$ of Eq. 11 on the interval $0 \leqslant t \leqslant t_{n}$, using some convenient initial conditions, such as

$$
p_{i}(0)= \begin{cases}\alpha_{2}, & i=1,2, \ldots, S, \\ 0, & i=S+1, \ldots, N\end{cases}
$$

Then we produce numerically $N-S$ independent vector solutions of the homogeneous equation

$$
h_{j}=J\left(x^{0}\right) h_{j}, \quad j=S+1, S+2, \ldots, N,
$$

where $h_{j}(t)$ is an $\lambda^{\prime}$-dimensional vector. For initial conditions we choose

$$
h_{j}(0)=\delta_{i j}, \quad j=\mathrm{S}+1, \mathrm{~S}+2, \ldots, N .
$$

Then the solution of Eq. 11 is representable in the form

$$
x^{1}(t)=p(t)+\sum_{i=1}^{x} c_{j} h_{j}(t)
$$

where $c_{s-1}, c_{s, 2} \ldots, c_{x}$ are constant $s$ to be determined. They are calculated as solutions of the linear algebraic equations

$$
\frac{\partial Q}{\partial c_{j}}=0, \quad j=S+1, S+2, \ldots, N,
$$

where for $x\left(t_{i}\right)$ we substitute

$$
x\left(t_{i}\right)=p\left(t_{i}\right)+\sum_{i=1}^{X} c_{j} h_{j}\left(t_{i}\right), \quad i=1,2, \ldots, M .
$$


In view of our choices of $p(0)$ and $h_{j}(0)$, we have as our new approximation to the initial vector

$$
x(0)=\left(\begin{array}{c}
\alpha_{1} \\
\vdots \\
\alpha_{S} \\
c_{S+1} \\
\vdots \\
c_{N}
\end{array}\right)
$$

The process can then be repeated to obtain an improved estimate. Detailed discussions and many other applications will be found in $[1]$.

NUMERICAL RESULTS

The quasilinearization technique was applied to the problem described in the Introduction. The results are shown in Table II.

TABLE II

NUMERICAL RESULTS

\begin{tabular}{crr}
\hline Approximation & \multicolumn{1}{c}{$k$} & \multicolumn{1}{c}{$K$} \\
\hline 0 & $1 \times 10^{-8}$ & $1 \times 10^{-4}$ \\
1 & $0.3413 \times 10^{-5}$ & $0.2554 \times 10^{-2}$ \\
2 & $0.4589 \times 10^{-5}$ & $0.3683 \times 10^{-3}$ \\
3 & $0.4578 \times 10^{-5}$ & $0.2808 \times 10^{-3}$ \\
4 & $0.4577 \times 10^{-5}$ & $0.2797 \times 10^{-3}$ \\
\hline
\end{tabular}

Further iterations produced no changes. The entire calculation consumed less then $30 \mathrm{sec}$ on an IBM 7044, no attempt having been made to streentline the calculations. By comparison, Bodenstein and Lindner, using a combination of chemical theory and the observations given earliur, estimated that

$$
\begin{aligned}
& k=0.53 \times 10^{-5}, \\
& K=0.41 \times 10^{-3},
\end{aligned}
$$

Mathenatical Biosciences 1, $71-76(1967)$ 
As a point of interest, the sum of the squares of the deviations for our choices of the parameters is $0.210 \times 10^{-2}$, and the sum of the squares of the deviations for Bodenstein's and Lindner's choice is $0.555 \times 10^{-2}$.

We do not imply that our values are more accurate than those of Bodenstein and Lindner. We only emphasize that our estimates were obtained in a systematic, computationally feasible manner requiring no further knowledge of the chemical process than that contained in Eq. 2, the observations, and the initial estimates.

\section{REFERENCES}

1 R. Bellman and R. Kalaba, Quisil'nearization and nonlinear boundary-value problems, American Elsevier, New York, 1965.

2 H. H. Kagiwada, R. E. Kalaba, and R. E. Bellman, Numerical estimation of devitatives with an application to radiative transier in spherical shells, The RAND Corporation, RM-4617-NASA, June, 1965.

3 R. E. Bellman and R. E. Kalaba, On a new approach to the numerical solution of a class of partial differential integral equations of transport theory, The RAND Corgoration, KM-4667.PR, August, 1965.

$4 \mathrm{R}$. Bellman, R. Kalaba, and M. Prestrud, Invariant imbedding and radiative iransfer in slabs of finile thickness, American Elsevier, New York, 1963.

¿ R. Bellman, H. Kagiwada, R. Kalaba, and M. Prestrud, Invariant imbedding and time-dependent transport processes, American Elsevier, New York, 1964.

6 R. Bellman, R. Kalaba, and J. Lockett, Numerical inversion of the Laplace ivansform with applications, American Elsevier, New York, 1966.

7 M. Bodenstein. Bildung und Zersetzung der Höheren Stickoxyde, Z. Physik. Chem. (Leipaig) 1100 (87). (1922), 88-123.

Mathemalical Biosciences 1, 71-76 (1967) 